\title{
Risk factors for the incidence and progression of radiographic osteoarthritis of the knee among Japanese
}

\author{
Akinobu Nishimura $\cdot$ Masahiro Hasegawa $\cdot$ Ko Kato $\cdot$ \\ Tomomi Yamada $\cdot$ Atsumasa Uchida $\cdot$ Akihiro Sudo
}

Received: 30 April 2010/Revised: 31 May 2010 /Accepted: 1 June 2010/Published online: 18 June 2010

(C) Springer-Verlag 2010

\begin{abstract}
This longitudinal study aimed to identify risk factors for the incidence and progression of radiographic knee osteoarthritis (OA). We examined the inhabitants of Miyagawa village aged $\geq 65$ years every two years between 1997 and 2007. Anteroposterior radiographs of both knees were graded for OA using the Kellgren-Lawrence $(\mathrm{K} / \mathrm{L})$ grading system. Knee OA was defined as grade $\geq 2$. We recorded the incidence of knee $\mathrm{OA}$ among participants in whom both knees changed from $\mathrm{K} / \mathrm{L}$ grades 0 or 1 to $\geq 2$ over a four-year follow-up period. We also recorded the progression of knee OA using this threshold among patients in whom one or both knees changed from $\mathrm{K} / \mathrm{L}$ grades 2 or 3 to any higher grade over the follow-up period. Baseline data obtained from standard questionnaires, physical findings and X-rays included age, gender, body mass index (BMI), osteoporosis, Heberden's nodes, knee range of motion (ROM), knee pain and cigarette smoking. The rates of incidence and progression of knee OA among 360 participants ( 241 women, 119 men) who fulfilled the study criteria were 4.0 and $6.0 \%$ per year, respectively. Female gender (odds ratio [OR] 2.849, 95\% confidence interval [CI] 1.170-6.944) and high BMI (OR 1.243, 95\% CI $1.095-1.411)$ were significantly associated with the incidence of knee OA, and restricted knee ROM (OR 0.941,
\end{abstract}

\footnotetext{
A. Nishimura $(\bowtie) \cdot$ M. Hasegawa $\cdot$ K. Kato $\cdot$ A. Uchida $\cdot$ A. Sudo Department of Orthopaedic Surgery,

Mie University Graduate School of Medicine,

2-174 Edobashi,

Tsu City, Mie 514-8507, Japan

e-mail: meiten@clin.medic.mie-u.ac.jp

T. Yamada

Department of Translational Medical Science,

Mie University Graduate School of Medicine,

Tsu, Mie, Japan
}

95\% CI 0.892-0.992) was significantly associated with knee OA progression. Patients with a low knee ROM relative to grade of radiographic knee $\mathrm{OA}$ require more careful follow-up than those with a higher ROM.

\section{Introduction}

Osteoarthritis (OA) of the knee is a major chronic disease among the elderly and an important cause of impairment and disability with associated health care costs [1]. Japanese society is rapidly aging and thus the incidence of Japanese patients with knee OA is increasing. To understand the risk factors affecting the incidence and progression of $\mathrm{OA}$ is very important for prevention, and to understand the ratio of affected individuals, the length of time that knee OA takes to develop and its frequency is imperative.

Several studies have described various risk factors and their associations with the incidence of knee OA [2-4]. We have also examined the prevalence and risk factors for knee OA in a cross-sectional study [5]. However, cross-sectional studies cannot address how risk factors affect the initiation or progression of knee OA, so longitudinal studies are needed to define effective prevention strategies. The risk factors involved in the progression and incidence of knee OA differ. For example, far more patients with, than without, knee OA undergo total knee arthroplasty, and the knee OA can remain stable over long periods or rapidly progress $[6,7]$. Conservative approaches such as pain medication and injections into the joint are more useful for low, than for high-grade (severe) knee OA. Severe knee OA often requires a surgical approach such as total knee arthroplasty. In order to prevent progression to severe knee $\mathrm{OA}$ and initiation of the disease are equally important 
public health strategies. Indeed, six of 1,172 participants in this study have already received total knee replacements. Thus, to distinguish progressive and non-progressive knee $\mathrm{OA}$ is important. However, few studies have attempted to differentiate incidence from progression in a longitudinal study [8-10]. We investigated risk factors for the incidence and progression of radiographic knee osteoarthritis among Japanese inhabitants of Miyagawa in a longitudinal study.

\section{Materials and methods}

The study participants were recruited from among inhabitants $\geq 65$ years of age in Miyagawa, a mountain village located in the centre of Mie Prefecture, Japan. The population of this village was 4,196 in 1997 , when 1,463 of residents met the age criterion. The population dropped to 3,624 by 2008 , at which time 1,544 individuals met the age criterion. We examined those who enrolled in 1997, 1999, 2001, and 2003 every two years, and those who enrolled during 2007, at Houtoku Hospital in the village. The Committee for the Ethics of Human Research of Mie University approved the study protocol, and all participants provided written, informed consent before enrollment in the study.

Baseline data obtained from standard questionnaires administered by orthopedic surgeons included information regarding age, gender, medical history, knee pain, and cigarette smoking. Knee pain was determined from the question, "Have you experienced knee pain lasting for over one month during the past year?" Knee pain was estimated as absent, one-sided or bilateral. The mean range of movement (ROM) of both knees was measured using a protractor. Height and weight were measured and then body mass index (BMI) was calculated. Other medical examinations comprised radiography of knees and hands (for Heberden's nodes) and bone mineral density (BMD). The BMD at the distal third of the non-dominant side radius was measured using dual energy X-ray absorptiometry (DCS600EX; Aloka, Tokyo, Japan). Osteoporosis was defined as 2.5 standard deviations (SD) of BMD lower than that of the young adult mean (YAM) of a healthy young adult of the same gender.

Fully extended anteroposterior (AP) radiographs of both knees (non weight-bearing) were scored for radiographic knee OA according to the Kellgren Lawrence $(\mathrm{K} / \mathrm{L})$ grading system [11]. Confirmed radiographic knee OA was defined as a $\mathrm{K} / \mathrm{L}$ grade of $\geq 2$. All knee radiographs were independently evaluated by three orthopaedic specialists and the final score was reached by consensus of two or three of them, but the middle score was accepted when the opinions of all three differed. A change in OA in both knees from a K/L grade of 0 or 1 to $\geq 2$ over a four-year follow-up period in individual patients was recorded as incidence. A change in OA in one or both knees from $\mathrm{K} / \mathrm{L}$ grades 2 or 3 at baseline to any higher grade over the follow-up period using this threshold was recorded as progression.

We used the grade of the most affected knee at baseline and follow-up to classify incidence and progression. Thus, only one knee per individual was analysed and generalised estimating equations were not required to account for correlations between both knees of each individual. Radiographically determined changes in either joint space or osteophyte scores were also analysed to derive estimates of incidence and progression. Individuals with rheumatoid arthritis or who had undergone total knee replacement were excluded. Rheumatoid arthritis was defined from answers to the question, "Have you ever been diagnosed with rheumatoid arthritis?" Ankylosing spondylitis was not identified in any of the participants.

Statistical analysis Means \pm standard deviations (SD) were calculated for variables unless otherwise noted. The risk of incidence and progression was analysed from multivariate logistic regression analysis. Variables included in the analysis were age, gender, BMI, osteoporosis, Heberden's nodes, ROM of bilateral knees, and knee pain (one or both knees). Results are summarised as odds ratios (OR) with 95\% confidence intervals (CI). The significance level for entry into the model was 0.05 . All data were statistically analysed using Stat View-J 5.0 software on a personal computer running Windows.

\section{Results}

Six patients who had received total knee replacements were excluded from among the 1,172 participants who attended six examinations associated with this study. We identified 360 villagers who fulfilled the study criteria. The main cause of exclusion was that many did not participate in the examination four years later. The cohort comprised 241 women and 119 men ranging in age from 65 to 89 years at baseline (overall mean age, $71.0 \pm 4.7$ years; $70.8 \pm 4.5$ and $71.3 \pm 5.1$ years for women and men, respectively).

Table 1 shows the distribution of $\mathrm{K} / \mathrm{L}$ grades for the most affected knee at baseline and at the four-year follow-up of the 360 participants. The prevalence of knee OA (Grade 2 or higher) increased from $27.5 \%$ (19.3\% male, $31.5 \%$ female) to $43.3 \%$ (30.3\% male, $49.8 \%$ female) over four years; that is, $4.0 \%$ of the cohort per year $(2.7 \%$ male, $4.6 \%$ female $)$ developed knee OA. The progression rate of knee OA was 22 (23.9\%) among 92 participants and $6.0 \%$ per year.

Table 2 shows relationships between each risk factor and the incidence of knee OA. Baseline $\mathrm{K} / \mathrm{L}$ grades of 0 or 1 
Table 1 Changes in radiographic Kellgren-Lawrence grades of knee osteoarthritis over four years in 360 participants

$O A$ osteoarthritis

Analysis based on worstaffected knee at baseline and follow-up

\begin{tabular}{lrrrrrrrl}
\hline Evaluation & 0 & I & II & III & IV & Total & \% of OA & \% of OA / year \\
\hline All & & & & & & & & \\
$\quad$ Baseline & 129 & 132 & 64 & 28 & 7 & 360 & 27.5 & \\
$\quad$ Follow-up & 75 & 129 & 96 & 48 & 12 & 360 & 43.3 & 4.0 \\
Male & & & & & & & & \\
$\quad$ Baseline & 47 & 49 & 14 & 6 & 3 & 119 & 19.3 & \\
Follow-up & 29 & 54 & 23 & 9 & 4 & 119 & 30.3 & \\
Female & & & & & & & & \\
$\quad$ Baseline & 82 & 83 & 50 & 22 & 4 & 241 & 31.5 & 4.6 \\
Follow-up & 46 & 75 & 73 & 39 & 8 & 241 & 49.8 & \\
\hline
\end{tabular}

advanced to grade $\geq 2$ in 57 of 261 participants (incidence OA group). This group significantly differed from the group without OA in terms of gender $(p=0.0209$; OR $2.849 ; 95 \%$ CI $1.170-6.944)$ and BMI $(p=0.0008$; OR $1.243 ; 95 \%$ CI 1.095-1.411)

Table 3 shows the relationship between each risk factor and progressive knee OA. Among 92 participants with baseline K/L grades 2 or 3, 22 of them advanced to a higher grade (Progressive OA group). This and the groups with no OA progression significantly differed in terms of knee ROM ( $p=0.0242$; OR, 0.941; 95\% CI, 0.892-0.992). Knee ROMs of $<120^{\circ}$ were identified in $7(31.8 \%)$ of 22 and in 5 (7.1\%) of 70 in the progressive and non-progressive OA groups, respectively.

\section{Discussion}

The results of this longitudinal cohort study of the inhabitants of Miyagawa showed that the rates of incidence and progression of knee OA were 4.0 and $6.0 \%$ per year, respectively. The risk of incidence of radiographic knee OA significantly increased among women with a higher baseline BMI. Risk for the progression of radiographically defined knee OA significantly increased when baseline knee ROM was lower.

The annual incidence rate of knee OA was $4.0 \%(2.7 \%$ male, $4.6 \%$ female), which is higher than previously reported. Zhang et al. [12] (Framingham study) found a rate of $1.9 \%$ (1.4\% male, $2.3 \%$ female) and Cooper et al. [13] found a rate of $2.5 \%$. We found that the rate of progressive knee OA was $6.0 \%$, which was also higher than that reported by Zhang et al. [12] and Cooper et al. [13], who reported rates of 3.6 and $3.7 \%$, respectively. We suppose that our results differed mainly because the age of our study cohort at baseline was $\geq 65$ years, whereas those of Zhang et al. [12] and Cooper et al. [13] were $>40$ and $>55$ years, respectively, which were relatively younger than our cohort. Race and lifestyle factors also differ. In general, our findings support the theory that advancing age increases the prevalence of knee OA.

Risk factors for the incidence of knee OA in many countries have been reported. They include obesity (high BMI) [14, 15], gender (female) [14], aging [14, 16], previous knee trauma [14], occupational kneeling or squatting [15, 17] and a higher BMD [18-20]. Obesity

Table 2 Determinants of incidence of radiographic knee osteoarthritis in 261 participants during the four-year follow-up period

\begin{tabular}{llllr}
\hline Demographic & Incident OA $(n=57)$ & No OA $(n=204)$ & Odds ratio & $95 \%$ CI \\
\hline Age & $70.8 \pm 20.4$ & $70.5 \pm 21.9$ & 1.021 & $0.953-1.094$ \\
Gender (female/male) & $44 / 13$ & $121 / 83$ & 2.849 & $1.170-6.944$ \\
BMI & $23.9 \pm 7.1$ & $22.6 \pm 6.3$ & 1.243 & $1.095-1.411$ \\
Osteoporosis (+/-) & $22 / 35$ & $73 / 131$ & 0.932 & $0.463-1.879$ \\
Heberden's nodes (+/-) & $29 / 28$ & $88 / 116$ & 1.307 & 0.0209 \\
Knee ROM & $141.4 \pm 65.5$ & $141.8 \pm 48.5$ & 1.009 & 0.0008 \\
Knee pain (-/+/++) & $34 / 14 / 9$ & $140 / 49 / 15$ & 1.434 & $0.965-1.056$ \\
Cigarette smoking (+/-) & $9 / 45$ & $48 / 159$ & 1.214 & $0.905-2.273$ \\
\hline
\end{tabular}

Knee pain defined as: - absent, + one sided, ++ bilateral

$O A$ osteoarthritis, $C I$ confidence interval, $B M I$ body mass index, $R O M$ range of movement 
Table 3 Determinants of progression of radiographic knee osteoarthritis in 92 participants during the four-year follow-up period

\begin{tabular}{|c|c|c|c|c|c|}
\hline Demographic & Progressive OA $(n=22)$ & Non-progressive OA $(n=70)$ & Odds ratio & $95 \% \mathrm{CI}$ & $P$ value \\
\hline Age & $70.7 \pm 4.7$ & $71.6 \pm 5.0$ & 0.934 & $0.826-1.055$ & 0.2706 \\
\hline Gender (female/male) & $3 / 19$ & $53 / 17$ & 1.318 & $0.223-7.752$ & 0.761 \\
\hline BMI & $25.4 \pm 4.7$ & $24.8 \pm 2.8$ & 0.932 & $0.779-1.114$ & 0.4372 \\
\hline Osteoporosis $(+/-)$ & $6 / 16$ & $20 / 50$ & 1.669 & $0.444-6.279$ & 0.4485 \\
\hline Heberden's nodes $(+/-)$ & $15 / 7$ & $38 / 32$ & 2.012 & $0.598-6.757$ & 0.2588 \\
\hline Knee ROM & $129.8 \pm 11.2$ & $136.8 \pm 11.3$ & 0.941 & $0.892-0.992$ & 0.0242 \\
\hline Knee pain $(-/+/++)$ & $7 / 10 / 5$ & $22 / 19 / 29$ & 0.628 & $0.316-1.250$ & 0.1851 \\
\hline Cigarette smoking $(+/-)$ & $2 / 20$ & $11 / 59$ & 0.732 & $0.087-6.151$ & 0.7742 \\
\hline
\end{tabular}

Knee pain defined as: - absent, + one sided, ++ bilateral

$O A$ osteoarthritis, $C I$ confidence interval, $B M I$ body mass index, $R O M$ range of movement

and previous knee trauma in Japan have also been reported as risk factors [2, 21, 22]. Our previous crosssectional study [5] also found that a higher BMI, female gender, more advanced age and a higher BMD were significantly associated with an increased risk of radiographic knee OA. This longitudinal study showed that gender and obesity were risk factors for the incidence of knee OA, whereas aging was not. Felson et al. [23] did not find an association between increased age and the incidence of OA, because their sample was elderly at study inception. Our study found no association between age and the incidence of knee OA. The incidence might level off in the elderly.

Understanding the risk factors for the progression and incidence of knee OA are equally important, because treatment differs according to grade. Conservative treatment (such as muscle-strengthening, supportive devices, physical therapy agents, non-steroidal anti-inflammatory drugs and intra-articular hyaluronic acid injections) are useful for mild (low-grade) knee OA; however, severe (high-grade) knee OA sometimes requires a surgical approach such as total knee arthroplasty. The risk factors for progressive knee OA reported by Felson et al. [8] are obesity, not smoking and weight gain, whereas those reported by Cooper et al. [13] are obesity, knee pain and Heberden's nodes. Felson et al. [5] reported that smoking favourably affects rates of other diseases, such as inflammatory bowel disease, and one of the many constituents of smoke might inhibit cartilage destruction. However, this finding is not universal and smoking did not affect the progression of knee OA in our study. Zhang et al. [12] reported that obesity and low BMD are risk factors for knee OA progression, whereas a high BMD is a risk factor for knee OA incidence. They indicated that only $\mathrm{BMD}$ as a risk factor differed between the progression and incidence of knee OA. We found here that a low knee ROM was the only risk factor for progressive knee OA. In particular, OA progressed more among participants with a knee ROM of $<120^{\circ}$ than among those with a knee ROM of $>120^{\circ}$. To our knowledge, knee ROM has not been examined, since low knee ROM has not been recognised as a risk factor for progressive knee $\mathrm{OA}$; yet restricting hip flexion by $>20 \%$ is a risk factor of progressive hip OA [24]. How low knee ROM causes knee OA to progress is not entirely clear. Nevertheless, the same mechanism involved in hip OA might also function in knee OA because the knees are also weight-bearing joints. We concluded that patients with a low knee ROM relative to grade of radiographic knee OA, particularly a low grade, require more careful follow-up.

Our study has several potential limitations. Firstly, Miyagawa is a mountain village, and many inhabitants are typically engaged in forestry. Secondly, participants who could attend the hospital were generally healthier than non-participants. Thirdly, this investigation was based on a relatively small cohort over a comparatively short duration. Therefore, the statistical significance of the risk factors might be relatively low. Further investigations are planned with more participants over a longer term, as the study will be continued every two years.

\section{Conclusion}

The incidence and progression of radiographic knee OA increased at rates of $4.0 \%$ and $6.0 \%$ per year, respectively, in a Japanese population aged $\geq 65$ years. Female gender and obesity (high BMI) were risk factors for incidence, whereas restricted ROM (low knee ROM) was a risk factor for the progression of knee OA. Patients with low knee ROM relative to grade of radiographic knee OA might require more careful follow-up than those with a higher ROM. 
Disclosure No benefits in any form have been received or will be received from a commercial party related directly or indirectly to the subject of this article.

\section{References}

1. Salaffi F, Carotti M, Stancati A, Grassi W (2005) Health-related quality of life in older adults with symptomatic hip and knee osteoarthritis: a comparison with matched healthy controls. Aging Clin Exp Res 17:255-263

2. Yoshimura N, Nishioka S, Kinoshita H, Hori N, Nishioka T, Ryujin M, Mantani Y, Miyake M, Coggon D, Cooper C (2004) Risk factors for knee osteoarthritis in Japanese women: heavy weight, previous joint injuries, and occupational activities. J Rheumatol 31:157-162

3. Hart DJ, Doyle DV, Spector TD (1999) Incidence and risk factors for radiographic knee osteoarthritis in middle-aged women: the Chingford study. Arthritis Rheum 42:17-24

4. Srikanth VK, Fryer JL, Zhai G, Winzenberg TM, Hosmer D, Jones G (2005) A meta-analysis of sex differences prevalence, incidence and severity of osteoarthritis. Osteoarthr Cartil 13:769-781

5. Sudo A, Miyamoto N, Horikawa K, Urawa M, Yamakawa T, Yamada T, Uchida A (2008) Prevalence and risk factors for knee osteoarthritis in elderly Japanese men and women. J Orthop Sci 13:413-418

6. Spector TD, Dacre JE, Harris PA, Huskisson EC (1992) Radiological progression of osteoarthritis: an 11 year follow up study of the knee. Ann Rheum Dis 51:1107-1110

7. Hernborg JS, Nilsson BE (1977) The natural course of untreated osteoarthritis of the knee. Clin Orthop Relat Res 123:130-137

8. Felson DT, Zhang Y, Hannan MT, Naimark A, Weissman B, Aliabadi P, Levy D (1997) Risk factors for incident radiographic knee osteoarthritis in the elderly: the Framingham Study. Arthritis Rheum 40:728-733

9. Slemenda C, Heilman DK, Brandt KD, Katz BP, Mazzuca SA, Braunstein EM, Byrd D (1998) Reduced quadriceps strength relative to body weight: a risk factor for knee osteoarthritis in women? Arthritis Rheum 41:1951-1959

10. Ledingham J, Regan M, Jones A, Doherty M (1995) Factors affecting radiographic progression of knee osteoarthritis. Ann Rheum Dis 54:53-58

11. Kellgren JH, Lawrence JS (1957) Radiological assessment of osteo-arthrosis. Ann Rheum Dis 16:494-502

12. Zhang Y, Hannan MT, Chaisson CE, McAlindon TE, Evans SR, Aliabadi P, Levy D, Felson DT (2000) Bone mineral density and risk of incident and progressive radiographic knee osteoarthritis in women: the Framingham study. J Rheumatol 27:1032-1037

13. Cooper C, Snow S, McAlindon TE, Kellingray S, Stuart B, Coggon D, Dieppe PA (2000) Risk factors for the incidence and progression of radiographic knee osteoarthritis. Arthritis Rheum 43:995-1000

14. Felson DT (1988) Epidemiology of hip and knee osteoarthritis. Epidemiol Rev 10:1-28

15. Coggon D, Croft P, Kellingray S, Barrett D, McLaren M, Cooper C (2000) Occupational physical activities and osteoarthritis of the knee. Arthritis Rheum 43:1443-1449

16. Felson DT, Naimark A, Anderson J, Kazis L, Castelli W, Meenan RF (1987) The prevalence of knee osteoarthritis in the elderly. The Framingham osteoarthritis study. Arthritis Rheum 30:914-918

17. Felson DT, Hannan MT, Naimark A, Berkeley J, Gordon G, Wilson PW, Anderson J (1991) Occupational physical demands, knee bending, and knee osteoarthritis: results from the Framingham study. J Rheumatol 18:1587-1592

18. Hannan MT, Anderson JJ, Zhang Y, Levy D, Felson DT (1993) Bone mineral density and knee osteoarthritis in elderly men and women. The Framingham study. Arthritis Rheum 36:1671-1680

19. Burger H, van Daele PL, Odding E, Valkenburg HA, Hofman A, Grobbee DE, Schutte HE, Birkenhager JC, Pols HA (1996) Association of radiographically evident osteoarthritis with higher bone mineral density and increased bone loss with age. The Rotterdam study. Arthritis Rheum 39:81-86

20. Sowers M, Lachance L, Jamadar D, Hochberg MC, Hollis B, Crutchfield M, Jannausch ML (1999) The associations of bone mineral density and bone turnover markers with osteoarthritis of the hand and knee in pre-and perimenopausal women. Arthritis Rheum 42:483-489

21. Yoshimura N, Kinoshita H, Hori N, Nishioka T, Ryujin M, Mantani Y, Miyake M, Takeshita T, Ichinose M, Yoshiida M, Oka H, Kawaguchi H, Nakamura K, Cooper C (2006) Risk factors for knee osteoarthritis in Japanese men: a case-control study. Mod Rheumatol 16:24-29

22. Iwamoto J, Takeda T, Ichimura S (2002) Forearm bone mineral density in postmenopausal women with rheumatoid arthritis. Calcif Tissue Int 70:1-8

23. Felson DT, Zhang Y, Hannan MT, Naimark A, Weissman BN, Aliabadi P, Levy D (1995) The incidence and natural history of knee osteoarthritis in the elderly. The Framingham osteoarthritis study. Arthritis Rheum 38:1500-1505

24. Reijman M, Hazes JM, Pols HA, Bernsen RM, Koes BW, BiermaZeinstra SM (2005) Role of radiography in predicting progression of osteoarthritis of the hip: prospective cohort study. BMJ 330:1183 